\title{
The potential of computer vision, optical backscattering parameters and artificial neural network modelling in monitoring the shrinkage of sweet potato (Ipomoea Batatas L.) during drying
}

\begin{abstract}
Background: Drying is a method used to preserve agricultural crops. During the drying of products with high moisture content, structural changes in shape, volume, area, density and porosity occur. These changes could affect the final quality of dried product and also the effective design of drying equipment. Therefore, this study investigated a novel approach in monitoring and predicting the shrinkage of sweet potato during drying. Drying experiments were conducted at temperatures of $50-70{ }^{\circ} \mathrm{C}$ and samples thicknesses of $2-6 \mathrm{~mm}$. The volume and surface area obtained from camera vision, and the perimeter and illuminated area from backscattered optical images were analysed and used to evaluate the shrinkage of sweet potato during drying. Results: The relationship between dimensionless moisture content and shrinkage of sweet potato in terms of volume, surface area, perimeter and illuminated area was found to be linearly correlated. The results also demonstrated that the shrinkage of sweet potato based on computer vision and backscattered optical parameters is affected by the product thickness, drying temperature and drying time. A multilayer perceptron (MLP) artificial neural network with input layer containing three cells, two hidden layers (18 neurons), and five cells for output layer, was used to develop a model that can monitor, control and predict the shrinkage parameters and moisture content of sweet potato slices under different drying conditions. The developed ANN model satisfactorily predicted the shrinkage and dimensionless moisture content of sweet potato with correlation coefficient greater than 0.95 . Conclusion: Combined computer vision, laser light backscattering imaging and artificial neural network can be used as a non-destructive, rapid and easily adaptable technique for in-line monitoring, predicting and controlling the shrinkage and moisture changes of food and agricultural crops during drying.
\end{abstract}

Keyword: Computer vision; Drying; Laser backscattering; Process control; Shrinkage; Sweet potato 